\title{
Conception d'interactions virtuelles et tangibles pour apprendre la collaboration motrice à des enfants avec Trouble du Spectre de l'Autisme
}

\author{
Design of virtual and tangible interaction to learn motor collaboration to children with ASD \\ Tom Giraud \\ ESTIA Recherche, Institut de \\ Technologie ESTIA, Bidart, \\ FranceUniversité Paris-Saclay, CNRS, \\ Laboratoire Interdisciplinaire des \\ Brian Ravenet \\ Université Paris-Saclay, CNRS, \\ Laboratoire Interdisciplinaire des \\ Sciences du Numérique, 91400, Orsay \\ FranceBrian.ravenent@limsi.fr \\ Jacqueline Nadel \\ TEDyBEAR: centre médico-social \\ autisme \\ Francej.nadel@centretedybear.com
}

Sciences du Numérique, 91400, Orsay

FranceTom.giraud.utc@gmail.com

\section{Brian Ravenet}

Université Paris-Saclay, CNRS,

Laboratoire Interdisciplinaire des

Sciences du Numérique, 91400, Orsay

FranceBrian.ravenent@limsi.fr

Gael Poli

TEDyBEAR: centre médico-social autisme

Franceg.poli@centretedybear.com

\author{
Chi Tai Dang \\ Human-Centered Multimedia Lab, \\ Augsburg University \\ Germanydang@informatik.uni- \\ augsburg.de \\ Elisabeth Andre \\ Human-Centered Multimedia Lab, \\ Augsburg University \\ Germanyandre@informatik.uni- \\ augsburg.de
}

\section{Elise Prigent}

Université Paris-Saclay, CNRS, Laboratoire Interdisciplinaire des Sciences du Numérique, 91400, Orsay

FranceElise.prigent@limsi.fr

Jean-Claude Martin

Université Paris-Saclay, CNRS,

Laboratoire Interdisciplinaire des

Sciences du Numérique, 91400, Orsay

Francejean-claude.martin@u-psud.fr

\begin{abstract}
New technologies for autism focus on training either social skills or motor skills, but never both. Such a dichotomy omits a wide range of joint action tasks that require the coordination of two people. Training these physical tasks performed in a dyad has great potential to promote inclusion, especially of children with autism spectrum disorders (ASD), including children who are nonverbal. In this article, we present the design of a tangible and virtual interactive system for training ASD children to perform joint actions. The proposed system consists of a virtual character projected onto a surface on which a tangible object is magnetized: both the user and the virtual character hold the object, thus simulating a joint action. Here, we present how the proposed interaction paradigm is innovative, while providing the results of a preliminary study with 10 typical children in order to prepare a long-term training with children with ASD.
\end{abstract}

This is the author's version of the work. It is posted here for your personal use. Not for redistribution. The definitive Version of Record was published in

IHM '20.21, April 13-16, 2021, Virtual Event, France

(C) 2021 Association for Computing Machinery.

ACM ISBN 978-1-4503-8362-2/21/04 ..\$15.00

https://doi.org/10.1145/3450522.3451335

\section{CCS CONCEPTS}

- Human-centered computing $\rightarrow$ Human computer interaction (HCI); Interaction paradigms; Mixed / augmented reality; Interaction design; Interaction design process and methods; User interface design.

\section{KEYWORDS}

Joint action, autism, tangible interaction, virtual agent

\section{RÉSUMÉ}

Les nouvelles technologies pour l'autisme se concentrent sur l'entraînement soit des aptitudes sociales, soit des aptitudes motrices, mais jamais les deux à la fois. Une telle dichotomie omet un large éventail de tâches d'action conjointe qui nécessitent la coordination de deux personnes. L'entraînement de ces tâches physiques effectuées en dyade a un grand potentiel pour favoriser l'inclusion, notamment des enfants avec troubles du spectre autistique (TSA) et non verbaux. Dans cet article, nous présentons la conception d'un système interactif tangible et virtuel pour la formation des enfants TSA à la réalisation d'actions conjointes. Le système proposé est composé d'un personnage virtuel projeté sur une surface sur laquelle un objet tangible est magnétisé : l'utilisateur et le personnage virtuel tiennent tous deux l'objet, simulant ainsi une action conjointe. Ici, nous présentons en quoi le paradigme d'interaction proposé est novateur, tout en fournissant les résultats d'une étude préliminaire avec 10 enfants typiques dans l'objectif de préparer un entraînement de long terme avec des enfants avec TSA. 


\section{MOTS-CLÉS}

Action conjointe, autisme, interaction tangible, agent virtuel

\section{ACM Reference Format:}

Tom Giraud, Brian Ravenet, Jacqueline Nadel, Brian Ravenet, Chi Tai Dang, Elise Prigent, Gael Poli, Elisabeth Andre, and Jean-Claude Martin. 2021. Conception d'interactions virtuelles et tangibles pour apprendre la collaboration motrice à des enfants avec Trouble du Spectre de l'Autisme: Design of virtual and tangible interaction to learn motor collaboration to children with ASD. In 32e Conférence Francophone sur l'Interaction Homme-Machine (IHM '20.21), April 13-16, 2021, Virtual Event, France. ACM, New York, NY, USA, 12 pages. https://doi.org/10.1145/3450522.3451335

\section{INTRODUCTION}

De nombreuses situations d'interaction avec des objets physiques nécessitent la collaboration d'une autre personne : un objet trop lourd pour être porté seul, un objet trop grand pour être tenu à deux mains, un objet trop glissant pour être tenu à deux bras... Comme de telles situations nécessitent la participation d'autres personnes, la question se pose de savoir de quoi sont capables des enfants atteints de Trouble du Spectre de l'Autisme (TSA) et présentant des difficultés motrices et des difficultés d'interaction sociale. Lorsque des individus se coordonnent pour réaliser des telles actions conjointes, les capacités sociales et motrices sont en jeu, de telle sorte qu'elles sont indissociables. Dans cette forme de "coopération incarnée" [1], le mouvement de l'autre offre de nouvelles possibilités pour nos propres actions [2]. Aider l'autre physiquement ne nécessite pas de se parler, et de ce fait un entraînement simulant une situation sociale de collaboration motrice peut être plus accessible aux personnes atteintes de TSA qui pour certaines n'ont pas accès au langage parlé. L'imitation synchrone est un des moyens le plus précoce, le plus simple et le plus efficace de partager un objectif moteur commun [3]. L'action conjointe pourrait donc être un objectif pertinent pour développer les capacités de collaboration des enfants atteints de TSA. Nous parlerons de "collaboration" plutôt que de "coopération". En effet, l'addition de deux motricités individuelles (coopération) ne suffit pas pour réussir une action motrice à deux (collaboration). S’il faut prendre en compte le mouvement de l'autre dans le contrôle de son propre mouvement (collaboration), un aspect social intervient sous la forme d'une synchronisation. Le système que nous avons conçu et que nous décrivons dans cet article vise ainsi à entraîner des enfants atteints de TSA à réaliser des actions conjointes, puis à long-terme à transférer ces apprentissages dans des collaborations motrices avec leurs pairs.

Comme les systèmes interactifs sont perçus par les enfants atteints de TSA comme des environnements stimulants, confortables et fiables [4], [5], de nombreux chercheurs ont conçu des interventions basées sur des technologies interactives (voir l'état de l'art cidessous). En ce qui concerne l'aspect spécifique de l'interaction sociale, les simulations informatiques offrent la possibilité de proposer une complexité sociale réduite et contrôlée. Cette complexité peut être progressivement augmentée tout au long de l'entraînement pour permettre un transfert en douceur vers les tâches du monde réel. Les compétences sociales sont généralement entraînées soit sur des tâches de communication via des robots ou des agents virtuels [6], soit sur des tâches de collaboration fine via des objets tangibles ou des tablettes [7].
Le système que nous avons conçu et que nous présentons dans cet article vise à entraîner de façon personnalisée des enfants atteints de TSA, y compris les enfants de type non verbaux, à réaliser des actions conjointes. L'approche sous-jacente pour ces enfants est que le moyen le plus simple et le plus direct d'améliorer l'engagement social est l'activité motrice. L'un des objectifs à long terme est d'encourager les enfants à participer à la vie familiale à la maison. Pour entraîner la collaboration motrice chez les enfants atteints de TSA, nous avons conçu une procédure ludique mais contrôlée expérimentalement. La procédure est implémentée dans une plateforme virtuelle composée d'objets mixtes, mi-virtuels et mitangibles, que l'enfant TSA doit déplacer à l'aide d'un agent virtuel. L'un des agents collabore avec l'enfant tandis que l'autre agent se comporte de manière autonome, ce qui oblige l'enfant à tenir compte du choix d'un objet par l'agent, du choix d'une trajectoire pour déplacer l'objet par l'agent et de la vitesse de déplacement de l'agent. Dans cet article, nous présentons la méthode de conception puis le système que nous avons conçu pour répondre à la problématique de l'entraînement à l'action conjointe d'enfants avec TSA ; nous décrivons ensuite les résultats d'une étude préliminaire de l'utilisation du système par des enfants typiques (étape intermédiaire dans le processus itératif de conception), et nous concluons par une discussion sur les étapes qui suivront ce travail en vue de l'utilisation du système avec des enfants atteints de TSA.

\section{ETAT DE L'ART}

\subsection{Interaction sociale médiée par ordinateur}

Une première approche en Interaction Humain-Machine (IHM) pour l'entraînement des enfants atteints de TSA aux compétences sociales est de concevoir des dispositifs structurants l'interaction sociale entre plusieurs enfants ou entre l'enfant et l'aidant. Dans la plupart des projets, le principal intérêt de ces dispositifs est de simplifier l'interaction sociale (en attribuant des rôles clairs ou en limitant les canaux de communications par exemple) tout en encourageant l'enfant à participer aux activités collaboratives.

L'interaction tangible fournit des interfaces physiques particulièrement adaptées aux contextes sociaux et collaboratifs, permettant à la fois un engagement corporel varié (offrant de multiples points d'entrée dans la situation interactive) et des contrôles partagés [8]. Des interfaces tactiles ont été conçues pour les enfants atteints de TSA afin de développer des compétences individuelles telles que la motricité fine [9] ou le jeu de simulation [10], avec la remarque récurrente par les chercheurs que les jouets physiques peuvent agir comme des facilitateurs sociaux. Dans le domaine des thérapies sociales pour les enfants atteints de TSA, un entraînement collaboratif basé sur les LEGO a été conçu pour améliorer la collaboration et les mécanismes de prise de rôle [11]. Un élément clé de cette thérapie est qu'elle simplifie l'interaction sociale en définissant explicitement le rôle de chaque enfant (un "ingénieur", un "constructeur" et un "fournisseur de ressources"). En suivant ce principe des thérapies de construction, Farr et ses collègues ont ainsi étudié les avantages de l'utilisation de jeux tangibles augmentés (d'abord avec le jeu Topobo@[12], ensuite avec un jeu de château physique augmenté [13]) où les mécanismes de rétroaction (visuels, kinesthésiques et sonores) rendent les objets plus attrayants et favorisent la compréhension des causes et des effets. 
Les mêmes conclusions ressortent de l'essai d'un entraînement aux activités collectives via la table interactive Reactable [14] (pour les performances musicales).

Des applications sur table interactives ont été développées pour les enfants atteints de TSA car elles combinent la flexibilité d'une interface numérique avec la dimension collective d'une table [15] Le projet SIDES propose un jeu coopératif où les enfants atteints de TSA devaient construire ensemble à tour de rôle un chemin avec des nénuphars [15]. Comme chaque joueur décide de la fin de son tour en appuyant sur un bouton, l'activité implique des compétences de négociation et de prise de tour de rôle. Un résultat intéressant de l'évaluation est que les enfants ont trouvé l'activité plus facile et plus relaxante avec des règles gérées par ordinateur. Dans le cadre du projet StoryTable [16], des dyades d'enfants atteints de TSA participent à l'écriture d'une narration collective. Certaines actions pouvaient être réalisées par un seul enfant, tandis que d'autres devaient être faites conjointement (par exemple, en touchant ensemble un bouton pour sélectionner un arrière-plan). Ce paradigme de collaboration forcée a montré qu'il favorise davantage d'initiations sociales et de jeux partagés après l'intervention. Ce type d'entraînement basé sur des gestes de coopération forcée a également été utilisé avec succès dans le développement de deux autres applications utilisant des tables : un puzzle collaboratif [17] conçu pour être résolu par une dyade d'enfants à travers une série de gestes coopératifs : se toucher, se déplacer et se relâcher ensemble ; et une série de trois mini-jeux [18] visant à former trois aptitudes sociales : l'action conjointe (via un jeu consistant à déplacer ensemble un panier pour attraper des pommes), le partage des ressources (via un jeu de construction de ponts en partageant des pièces complémentaires) et la planification mutuelle (via un jeu de collecte d'étoiles, un enfant détachant les étoiles tandis que l'autre les attrape avec un panier). Dans les deux cas, l'évaluation a montré que la condition de collaboration forcée induisait davantage de mouvements de coordination pour les enfants atteints de TSA.

Si ces projets d'interactions tangibles et de tables interactives montrent l'intérêt d'une interaction sociale structurée et rendue attractive ou forcée, ils restent limités sur deux aspects : l'interaction reste complexe pour des enfants atteints de TSA sévère de types non-verbaux, et la dimension motrice de l'entraînement n'est que très peu considérée.

\subsection{Entrainer des compétences non verbales avec des agents incarnés}

L'expressivité et l'interactivité contrôlées des agents incarnés, qu'il s'agisse d'agents virtuels ou de robots, permettent aux chercheurs de développer des applications adaptées à la formation de compétences sociales spécifiques. En raison de leur présence physique, les robots sont de puissants attracteurs et peuvent profiter de cette attention pour engager l'enfant dans des activités interactives. Les compétences d'imitation, avec leur dimension sociale et motrice, ont été formées par des robots sociaux [19]. Robota et Nao sont deux robots populaires utilisés dans ces thérapies d'imitation, où des mouvements et des postures simples des bras doivent être suivis ou initiés [20], [21], ce qui induit une imitation plus spontanée chez les enfants atteints de TSA [22]. Des résultats similaires ont été obtenus avec Tito, un robot ressemblant à une marionnette [23].
Des modèles interactifs plus complexes combinant l'imitation et les mécanismes d'attention conjointe ont été développés [24].

Les agents virtuels ont été principalement développés pour entraîner la communication affective [25], [26] et l'attention conjointe [27], [28], intégrés dans des jeux sérieux. JeMime est un jeu destiné à entraîner les compétences de production d'émotions [26]. L'idée du projet est de guider la production d'expressions faciales pertinentes (grâce à des jauges) en fonction de la situation sociale. Le projet ASC-Inclusion propose un jeu qui comprend à la fois des tâches de reconnaissance d'émotions et d'expressions pour le visage, le corps et la voix [29]. Dans le projet ECHOES, l'agent virtuel pouvait agir sur l'environnement virtuel, pointer des objets et orienter son regard pour initier une attention conjointe [30]. À ce jour, les agents incarnés ont été développés pour former les compétences sociales distales avec quelques succès, mais aucun projet n'a été développé pour la formation de l'action conjointe dans ses dimensions sociales et motrices. Les principales études existantes sont limitées à la coopération (chacun utilise ses propres mouvements indépendamment) et non à la collaboration.

\subsection{Des agents incarnés pour l'entrainement à l'action conjointe d'enfant atteint de TSA ?}

Comme précisé dans l'introduction, l'action conjointe, définie comme la collaboration motrice en vue de manipuler un objet commun, a le potentiel d'offrir une porte d'entrée accessible (via la motricité) dans la sphère du sociale pour des enfants atteints de TSA [31]. La simulation de l'action conjointe est un domaine actif de la robotique moderne [32] (figure 1), où les enjeux d'assignement de rôles et de dynamiques collaboratives sont débattus [33]. Ces travaux restent actuellement à l'état de recherche et sont donc loin d'être transférables dans un contexte sensible comme celui d'une tâche avec en enfant atteint de TSA.

La majeure partie des travaux en robotique sociale à destination d'enfants atteints de TSA garde à distance les robots, ceux-ci remplissant en premier lieu une fonction de communication. Deux projets intègrent la possibilité d'un engagement physique entre le robot et les enfants atteints de TSA. Le robot Probo [35] (figure 2 , gauche), un robot «attrapable », a la possibilité d'exprimer des émotions, divers comportements non-verbaux et son enveloppe a été pensée pour avoir une apparence chaleureuse encourageant l'étreinte, notamment via son revêtement de mousse et de coton similaire à celle d'une peluche éléphant. Pour permettre à des enfants d'interagir avec un robot dans un contexte terrain sensible, la partie hardware a fait l'objet d'un travail de recherche à part entière [36]. Ainsi, l'ensemble des actionneurs du dispositifs ont été mis en série avec des ressorts pour apporter de l'élasticité au robot en cas de collision et offrir une expérience de «toucher doux »[37]. Très récemment, le projet CASTOR (CompliAnt SofT Robotics) a développé un robot (figure 2, droite) aussi basé sur les principes de la «robotique douce »(soft robotics) en vue de permettre la mise en place de thérapies sociales en contexte réel et avec engagement physique [38]. Si la robotique douce ouvre la porte des thérapies motrices et sociales pour des enfants atteints de TSA, elle n'en est qu'à ses débuts et l'élasticité inhérente de ces robots semblent aller en contradiction avec le besoin de co-manipulation d'une action conjointe. 

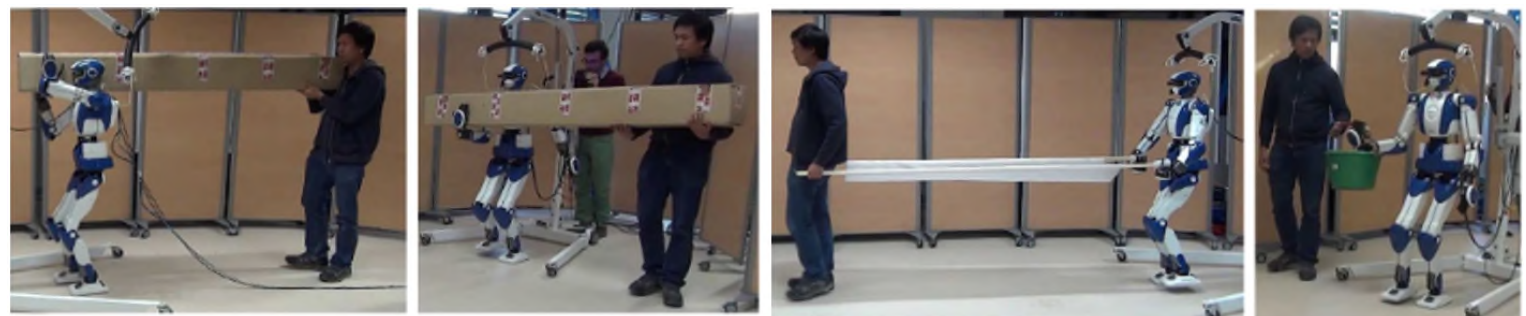

Figure 1: Quatre tâches motrices collaboratives [34]
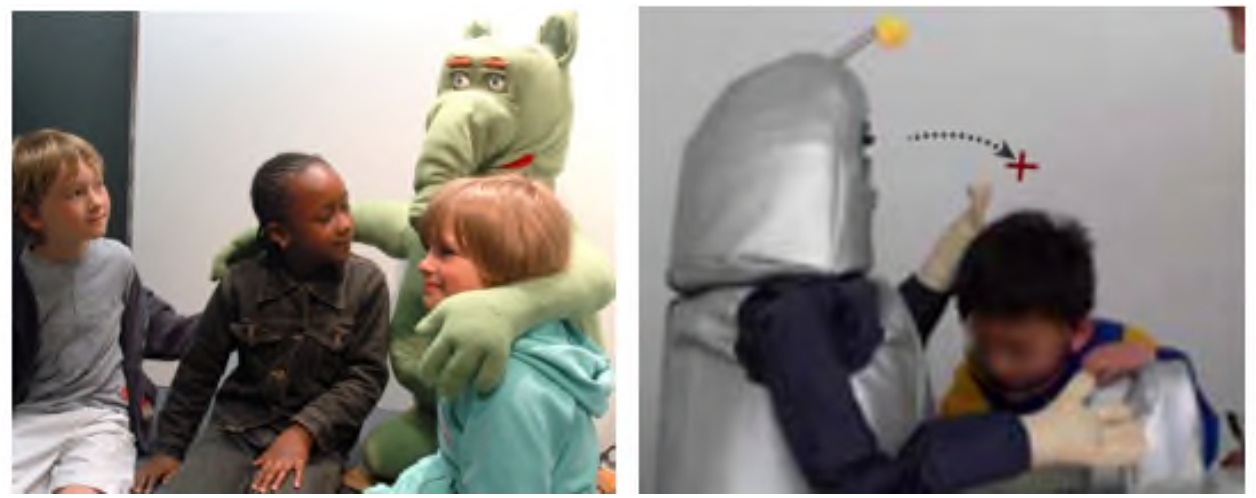

Figure 2: Gauche : le robot PROBO [35]. Droite : le robot CASTOR [38].
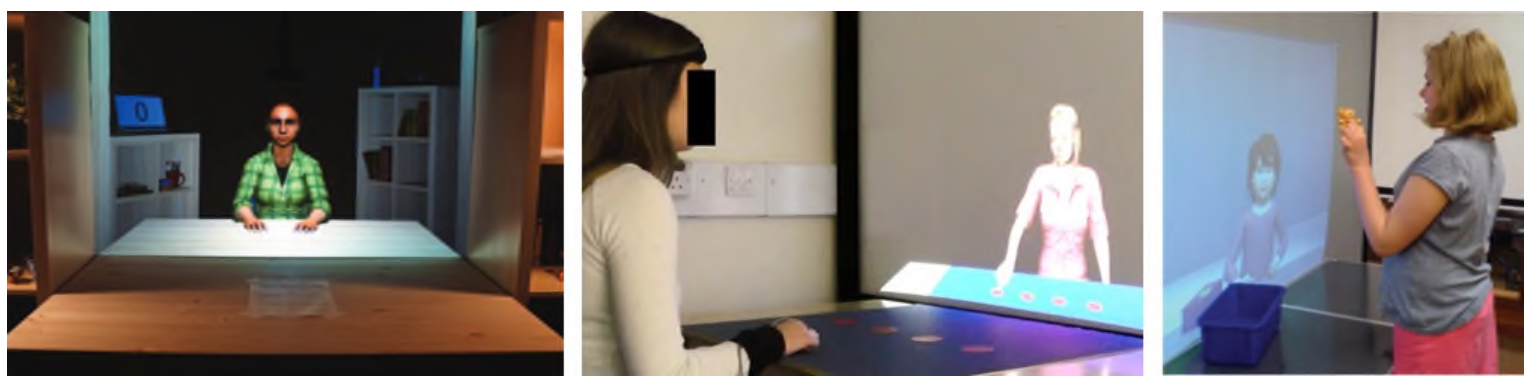

Figure 3: Gauche : Wobbly table [39]. Centre : tâche d'imitation [40]. Droite : tâche de jeu en miroir [41].

Une piste pour garder la dimension incarnée de l'agent et permettre une activité physique collaborative est d'augmenter physiquement l'affichage d'un agent virtuel. C'est ce qui a été fait lors du projet de la «Wobbly table »(figure 3, gauche) ayant pour objectif d'augmenter l'effet de présence sociale par le partage entre l'utilisateur et l'agent d'une table mi tangible - mi virtuelle [39]. Ce type de paradigme de la fenêtre réelle - virtuelle a par la suite été utilisé lors d'une tâche d'imitation entre des adultes atteints de TSA et un agent virtuel [40] (figure 3, centre), ainsi que lors d'une tâche de jeu en miroir entre un enfant atteint de TSA et un agent virtuel [41] (figure 3, droite).

Ces différents projets n'utilisent la dimension physique que comme un élément de continuité entre le réel et le virtuel, mais présentent l'avantage d'être intrinsèquement sécuritaire pour le contexte sensible d'une tâche avec un enfant atteint de TSA : la technologie se retrouve cachée derrière l'écran. Un des objectifs de ce projet a été d'explorer les possibilités du concept de l'humain virtuel augmenté en vue de proposer un entraînement à l'action conjointe sécuritaire et évolutif. Dès lors, l'enjeu principal d'un tel projet devient : comment permettre à un enfant d'avoir la sensation de tenir un objet physique avec un agent virtuel ?

\section{METHODE}

\subsection{Contexte du projet}

La présente recherche a été menée par une équipe interdisciplinaire comprenant des chercheurs en IHM et en psychologie, ainsi que des chercheurs et des praticiens en psychopathologie du développement travaillant dans un centre d'accueil pour enfants avec autisme. L'équipe de psychopathologie du développement a une longue expérience de recherche dans le développement des compétences 
d'imitation des enfants atteints de TSA [caché]. L'équipe expérimente également l'utilisation thérapeutique du jeu PictogramRoom basé sur Kinect [42], [43]. Cette pratique a permis à l'équipe d'acquérir de solides connaissances sur la manière de concevoir et de mettre en œuvre des thérapies interactives basées sur le corps pour les enfants atteints de TSA.

\subsection{Principes pour l'entrainement à l'action conjointe}

Lors des thérapies d'imitation synchrone, chaque partenaire négocie le rythme avec l'autre afin de réaliser une co-action, en alternant les rôles d'initiateur et d'imitateur [44]. Dans ce cas, les partenaires développent leurs propres gestes sans interférer physiquement avec les gestes de l'autre. Un autre point d'entrée dans le domaine social est la réalisation collaborative d'actions physiques (c'est-àdire d'actions conjointes). En fondant la formation des compétences sociales sur des actions physiques concrètes, on peut trouver un moyen plus direct de parvenir à une collaboration avec les autres. Le but de notre projet est donc d'entraîner les compétences motrices sur des actions physiques qui pourraient progressivement (en trois phases) devenir des actions conjointes nécessitant la collaboration motrice d'un partenaire :

- Phase 1 : entraînement à l'autonomie motrice pour permettre aux participants de réaliser seuls les mouvements nécessaires à la tâche.

- Phase 2 : entraînement à l'action conjointe avec un agent virtuel coopératif qui se synchronise avec les mouvements de l'enfant.

- Phase 3 : entraînement à l'action conjointe avec un agent virtuel autonome qui demande à l'enfant d'adapter sa motricité.

Notre approche vise à développer un système d'entraînement qui permet non seulement de suivre cette approche par phase, mais aussi de prendre en compte la diversité des enfants atteints de TSA et leurs spécificités individuelles [45]. Le système développé doit être accessible à des enfants montrant différents niveaux de troubles cognitives, motrices, verbaux et non-verbaux, afin d'éviter de se focaliser uniquement sur un autisme à haut fonctionnement. C'est pourquoi la cible première du projet sont des enfants (entre 4 et 10 ans) atteints de TSA et non-verbaux. Le cœur du projet, l'entraînement de compétence sociales à travers l'action conjointe va dans le sens de notre démarche d'inclusivité. Un autre élément qui va dans ce sens est la personnalisation de l'entraînement au plus près du besoin de l'enfant. Suivant l'idée que "chaque enfant est unique", le projet a pour ambition d'offrir un entraînement flexible plutôt que standardisé, dans lequel le praticien est capable d'adapter dynamiquement la tâche et la difficulté de celle-ci à la performance de l'enfant.

\subsection{Processus de conception}

Le processus de conception que nous avons suivi se décompose en plusieurs phases. Le projet a débuté par une phase de recherche de conception visant à préciser à la fois le contenu de l'entraînement et la conception du système interactif. Elle comprenait tout d'abord une phase d'observation participante au sein d'un centre pour enfants atteints de TSA (dont les âges devaient se situer entre 4 et 10 ans), phase qui nous a permis de relever un ensemble d'éléments utiles à la conception du dispositif :

- Les praticiens adaptent chaque activité à chaque enfant

- Les praticiens font les activités avec les enfants (parfois en prenant leur place)

- Les praticiens sont extrêmement attentifs à l'enfant (et ne veulent pas être dérangé par la technique)

- Les objets tangibles sont doux, robustes, et variés pour éviter les intérêts restreints

- Les objets tangibles sont le support de diverses activités collaboratives

Dans un deuxième temps, nous avons mis en place une série de séances d'idéations avec les praticiens impliqués dans le projet afin de préciser de manière itérative les différents éléments du système. Cette phase comporte 9 rencontres étalées sur 9 mois afin d'explorer et débattre d'alternatives de conception. On peut synthétiser les discussions menant aux différents choix techniques autour de trois axes :

- Innocuité du dispositif : ce critère central au sein des centres d'accueil a eu pour conséquence d'écarter la piste robotique et de mettre en avant la proposition d'une interaction avec agent virtuel. Le principe d'une paroi réel-virtuelle avec manipulation d'un objet tangible en mousse aimantée est aussi issu de ces débats.

- Réalisme de la tâche : pour assurer une plus grande transférabilité des acquis de l'entraînement à la vie quotidienne, il a été décidé de se rapprocher autant que possible des codes d'une interaction réelle. Ainsi la paroi réelle-virtuelle a été réalisée comme une fenêtre, et les objets tangibles conçus pour avoir les comportements d'objets réels.

- Scénarios et dialogue moteur : la complexité de l'entraînement à l'action conjointe devait pouvoir évoluer aussi bien au niveau moteur qu'au niveau social. Au niveau moteur, quatre schèmes moteur ont été définis (poser, lever, tirer, pousser) et une tâche de rangement d'objets (similaire à l'action de ranger sa chambre par exemple) conçue comme une composition de ces schèmes moteurs. Au niveau social, deux rôles d'agents ont été conçus : un agent coopératif (qui suit l'enfant) et un agent indépendant (que l'enfant doit suivre). Une complexité supplémentaire pour l'agent indépendant est qu'il peut effectuer la tâche à 3 vitesses différentes : la vitesse de confort de l'enfant, plus vite que l'enfant, et plus lentement que l'enfant.

Cette phase a abouti à un prototype fonctionnel décrit dans la section suivante sur la réalisation du système. La phase d'expérimentation prévue dans le projet se compose d'une étude en laboratoire avec des enfants typiques afin de recueillir des informations préliminaires sur la validité du paradigme d'interaction, d'un entraînement expérimental sur le terrain avec de jeunes enfants atteints de TSA afin de tester l'appareil dans des conditions réelles, et enfin d'un entraînement expérimental sur le terrain avec de jeunes adolescents atteints de TSA afin d'explorer le potentiel du dispositif pour d'autres types de publics. Dans cet article, nous présentons les résultats préliminaires de la première étude. 


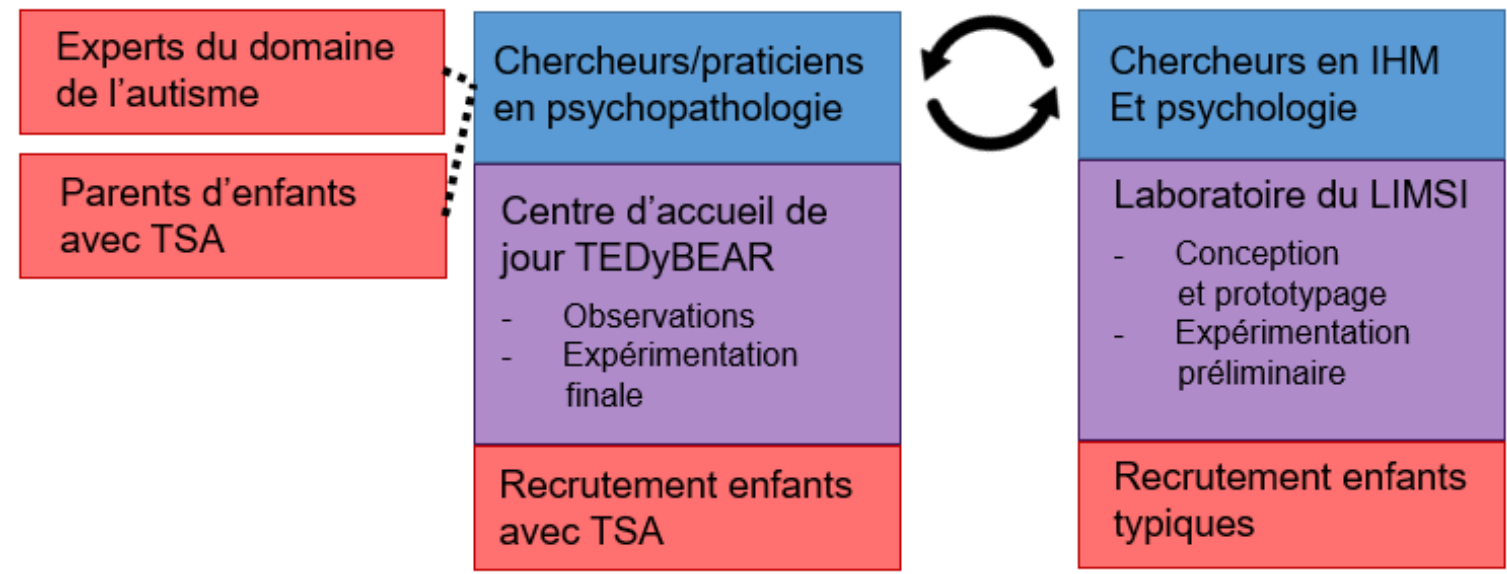

Figure 4: Schéma des acteurs impliqués dans le projet.
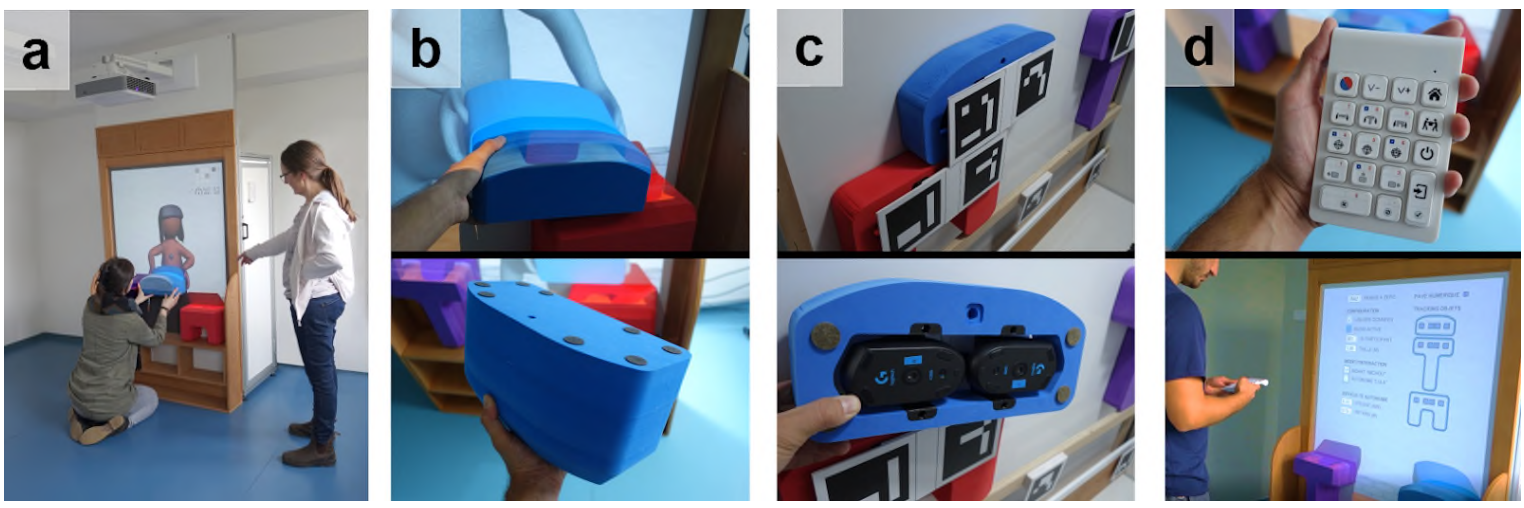

Figure 5: a. Installation interactive complète. b. Objet tangible sur le mur et détaché de celui-ci. c. Système de suivi intégré à l'objet jumeau arrière. $d$. Télécommande pour configurer la formation.

\section{DESCRIPTION DU SYSTÈME}

\subsection{Le paradigme d'interaction}

Le système proposé vise à simuler une action conjointe entre un enfant et un personnage virtuel (figure 5.a). Il est composé d'un personnage virtuel projeté sur une surface verticale sur laquelle un objet tangible est magnétisé : l'enfant et le personnage virtuel tiennent et déplacent tous deux l'objet, ce qui permet de simuler une action conjointe. L'enfant peut tenir et déplacer l'objet tangible. Le personnage virtuel peut déplacer ce qui ressemble à la partie graphique de l'objet tangible. Ce système est basé sur le paradigme d'interaction de la fenêtre réelle-virtuelle [46] : le mur projeté est une fenêtre sur l'espace virtuel du personnage virtuel supposé être la continuité de l'espace réel du participant, les participants peuvent "voir à travers" le mur.

\subsection{Structure du dispositif}

La figure 5.a montre la structure du dispositif avec trois objets tangibles interactifs positionnés sur celui-ci. Le dispositif se compose d'une structure en aluminium supportant la fenêtre interactive $\left(1,5 \mathrm{~m}{ }^{*} 1 \mathrm{~m}\right)$, d'une structure en bois conçue comme une façade de mobilier et un cadre pour la fenêtre, d'un vidéoprojecteur, et d'une face arrière $(0,6 \mathrm{~m}$ de profondeur) fermée par une porte avec tout le matériel nécessaire à l'intérieur. L'appareil est stable et résiste aux coups sans aucun risque. Comme l'appareil est imposant, la façade en bois apporte une certaine familiarité et la couleur blanche de la partie supérieure de l'appareil limite sa saillance dans le champ perceptif. Une vidéoprojection à très courte portée a été choisie pour limiter les ombres pendant l'interaction.

\subsection{Objets interactifs}

4.3.1 Matériaux et glissement. Les objets tangibles sont fabriqués sur mesure en EVA, une mousse de haute densité utilisée pour les jeux d'enfants et les tapis. Dans ce projet, le choix a été fait de concevoir trois objets représentant une table (en violet), un tabouret (en rouge) et une boîte (en bleu). Chaque objet tangible tient sur le mur car il est magnétisé à un objet jumeau situé derrière le mur (figure 4.b \& 4.c). Pour permettre aux parties avant et arrière des objets de glisser facilement tout en étant fermement attirées l'une vers l'autre, le mur est constitué d'un matériau composite mince (4 mm d'Alu Dibond®) recouvert d'un revêtement glissant sur les deux faces (Velleda@). Des aimants de différentes puissances 
ont été testés pour trouver le meilleur compromis entre une forte attraction et un bon glissement. Des chanfreins ont été ajoutés aux objets frontaux pour inciter la saisie de l'objet au plus près du mur, ce qui limite le risque de détachement accidentel. Cette solution a été retenue plutôt qu'un système similaire à Reactable car dans notre cas, il était nécessaire d'avoir une paroi verticale et opaque pour cacher les objets jumeaux de l'autre côté de la paroi, qu'un rétro-éclairage aurait mis en avant.

4.3.2 Suivi des objets. Lorsque l'enfant fait glisser l'objet de devant le long du mur, l'objet jumeau correspondant caché derrière le mur suit. Grâce à ce principe, le suivi peut être intégré dans l'objet jumeau et être caché à l'enfant. Le choix du système de suivi a été guidé par trois facteurs (par ordre d'importance) :

- Fiabilité : le paradigme de l'interaction des fenêtres repose sur la continuité robuste entre la partie virtuelle et la partie réelle de l'objet tangible. Un aspect critique était de concevoir une solution de suivi offrant un délai minimal pour donner l'impression que la partie virtuelle et la partie tangible "se collent" et forment le même objet

- Compacité : le système doit pouvoir être installé à l'arrière de l'appareil

- Reproductibilité : l'appareil est conçu pour être reproductible et facile à entretenir

Afin de respecter ces facteurs et d'assurer en même temps que le coût des composants du système ne devienne pas un frein à sa réplication, le suivi 2D en temps réel de l'objet tangible est réalisé par la combinaison des deux systèmes suivants (figure 5.c) :

- Un suivi relatif et à faible retard réalisé avec deux souris intégrées. En plus de leur grande réactivité, les souris sont compactes et très fiables.

- Un suivi absolu basé sur des marqueurs, réalisé à l'aide d'une webcam et de deux marqueurs Aruco sur la partie jumelle de chaque objet situé de l'autre côté de la paroi. Le suivi continue donc même lorsque l'objet est décroché. Ceci permet au praticien (ou à l'enfant) de savoir où il doit repositionner l'objet pour l'aimanter à nouveau avec sa partie jumelle sans avoir à ouvrir le dispositif. Ce système a été ajouté en complément du suivi par la souris pour corriger la dérive accumulée.

La fusion de ces données en temps réel est réalisée en fonction de la vitesse de déplacement : les données de suivi des souris sont utilisées au-dessus d'un seuil de vitesse et les données de suivi des marqueurs sont utilisées en dessous, corrigeant la dérive accumulée pendant les phases de déplacement rapide.

\subsection{Personnages virtuels interactifs}

4.4.1 Morphologie et comportements. La partie logicielle de l'appareil a été conçue avec le moteur de jeu Unity. Les personnages virtuels anthropomorphes ont été conçus sans caractéristiques et expressions faciales pour limiter la complexité sociale de la scène virtuelle. Comme l'entraînement implique d'interagir dans deux modes (un mode suiveur et un mode leader), deux personnages virtuels différents ont été conçus, différenciés l'un de l'autre par une couleur et un chapeau différent (figure 6.a). Attribuer un rôle spécifique à un personnage identifiable permet de simplifier l'interaction sociale et d'apporter une variabilité attrayante à l'entraînement. Des noms ont été donnés à chaque personnage pour les personnifier et faciliter les instructions orales pour les praticiens ("Michou" pour le personnage suiveur, "Lola" pour le personnage meneur).

Les comportements non verbaux de l'agent ont été conçus de manière à être aussi simples que possible. Lors des phases inactives, le personnage est animé par une animation d'attente. À la demande du praticien, le personnage virtuel peut saisir un objet virtuel. Cette animation de saisie a été réalisée à l'aide d'un algorithme de cinématique inverse humanoïde. Deux modes d'interaction ont été conçus

- Mode suiveur : le personnage tient l'objet virtuel qui reste aligné sur la partie tangible. L'enfant doit effectuer un mouvement vers une cible spécifique indiquée par le praticien. Dans la figure 6.b, l'instruction est de déplacer avec Michou la boîte bleue de la table violette vers le tabouret rouge.

- Mode meneur : le personnage initie un mouvement avec la partie virtuelle de l'objet qui se détache visuellement de la partie tangible de l'objet. L'enfant doit suivre le mouvement du personnage vers une cible spécifique qui lui est inconnue. Si l'enfant ne suit pas l'objet virtuel d'assez près, le personnage principal libère l'objet et une animation de fumée s'affiche autour de la partie virtuelle de l'objet. Dans la figure 6.c, l'instruction est de se déplacer avec Lola la boîte bleue en suivant Lola (sans spécifier la destination).

4.4.2 Contrôles et retour d'information. Pour chaque session d'entraînement, un scénario est défini comme la tâche consistant à déplacer un objet spécifique vers une cible spécifique en même temps que le personnage virtuel. Le praticien contrôle les types et la quantité de scénarios qu'il veut exécuter avec l'enfant. Un scénario est automatiquement considéré comme un succès (avec des étoiles affichées et tournant autour de la partie virtuelle de l'objet, figure 7, gauche) lorsque l'objet tangible tenu par l'enfant atteint la cible. Il est automatiquement considéré comme un échec (avec une animation de fumée douce, figure 7, droite) si l'enfant ne réussit pas à suivre correctement le personnage principal. En plus de ces décisions automatiques, le praticien peut prendre la main sur celles-ci et étiqueter manuellement un scénario comme étant un succès, un échec ou un scénario avorté. Cette flexibilité est importante pour leur permettre d'expérimenter le dispositif et d'adapter l'évaluation de la formation à chaque enfant. Les praticiens peuvent également configurer la session via un écran d'accueil (retour audio, hauteur, mode interactif, vitesse, seuil d'échec).

Pendant une séance, les praticiens doivent rester attentif et engagé dans l'interaction avec l'enfant et éviter toute distraction. Nous avons donc conçu une télécommande légère et personnalisée pour manipuler l'appareil (figure 5.d). En utilisant un pavé numérique sans fil recouvert d'icônes personnalisées, les praticiens peuvent configurer la session via l'écran d'accueil et contrôler les scénarios durant d'interaction. La télécommande peut être utilisée en la tenant dans la main ou en la plaçant dans son support personnalisé sur le côté de l'appareil.

4.4.3 Visualisation des séances. Le dernier composant du système est un ensemble d'outils permettant aux praticiens de visualiser et 

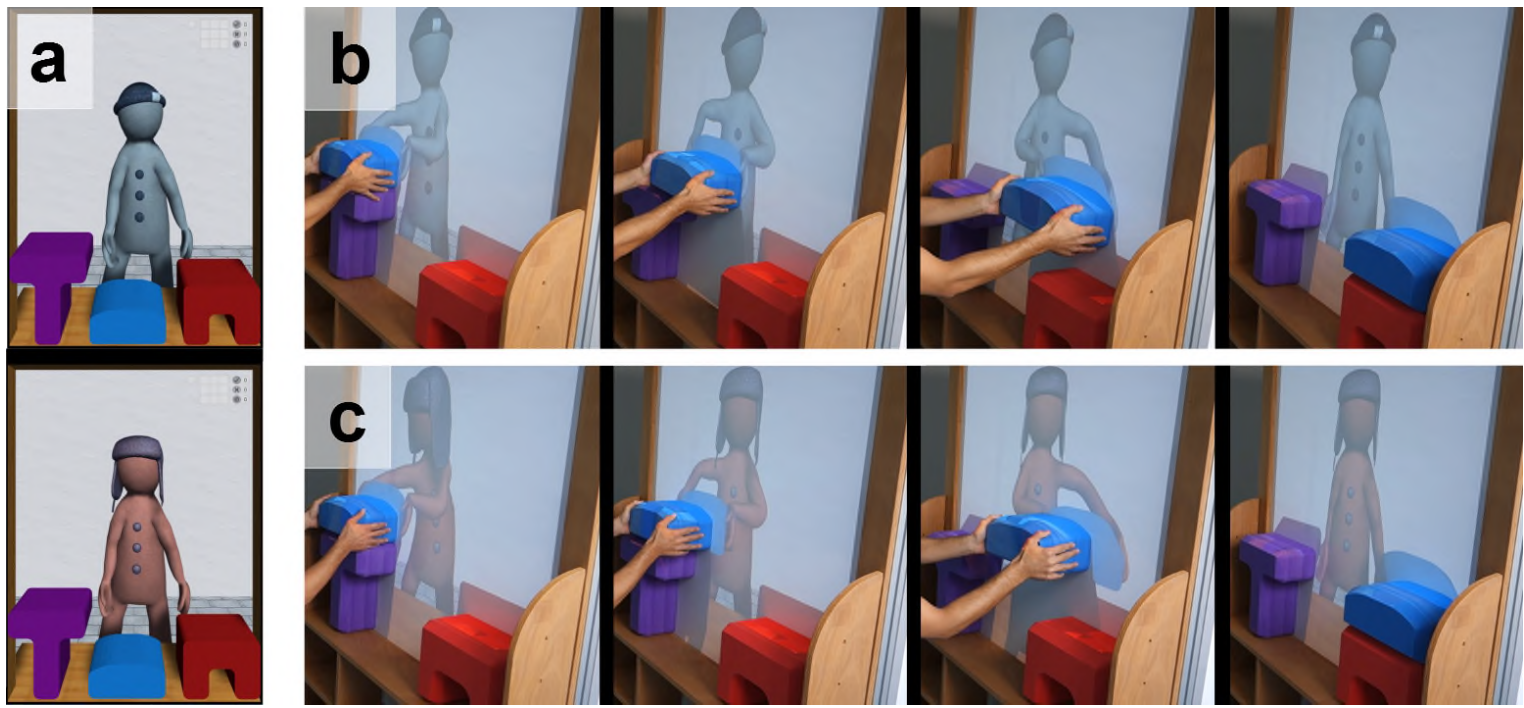

Figure 6: a. Les deux agents virtuels. b. Séquence de mouvements en mode suiveur. c. Séquence de mouvements en mode meneur.
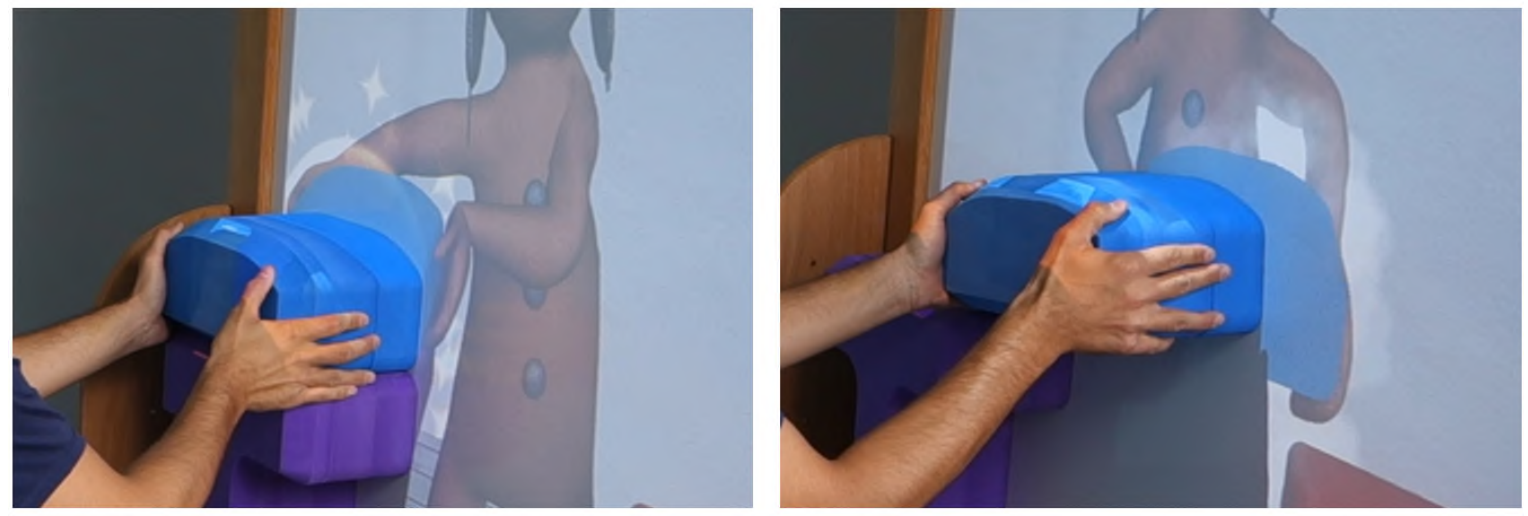

Figure 7: Gauche : animation de succès (étoiles autour de l'objet). Droite : animation d'échec (fumée autour de l'objet).

de revenir sur les interactions. Toutes les interactions avec le système sont enregistrées par celui-ci. Les données qui sont conservées sont pour chaque séance, l'ensemble des paramètres des séances (date, configuration, mode de l'agent) ainsi que les mouvements effectués par les objets durant chaque scénario réalisé par l'enfant. Il est bien sûr à préciser que le système ne peut suivre que les parties des objets qui se trouvent de l'autre côté de la paroi, celles qui servent au suivi à l'aide des souris et des marqueurs Arucos et que c'est en réalité leurs déplacements qu'ils enregistrent. Si l'enfant décroche sa partie de l'objet, le système ne le sait pas et continue d'enregistrer les déplacements des parties suivies.

Le système fournit des outils permettant de traiter ces données afin d'en extraire des informations descriptives sur les séances (e.g. vitesse moyenne des déplacements, écart moyen avec l'agent), ainsi que des images et des vidéos rejouant les déplacements effectués par l'enfant, permettant aux praticiens de revoir a posteriori certaines séances et de visualiser sous une autre forme les performances des enfants (voir figure 8). En particulier, ces outils permettent de visualiser les écarts spatiaux et temporels entre les trajectoires de l'agent et celles de l'enfant. La trajectoire de l'agent est représentée sous la forme d'un trait noir et la trajectoire de l'enfant est représentée sous la forme d'un trait de la couleur de l'objet qu'il déplace (le trait est plus clair au début du mouvement, plus foncé à la fin). L'épaisseur du trait dépend de la vitesse de l'enfant. De fines lignes noires entre la trajectoire de l'enfant et celle de l'agent indiquent le lien temporel entre les trajectoires, permettant de visualiser si l'enfant anticipait ou était en retard sur le mouvement de l'agent. Cette visualisation graphique permet ainsi une analyse personnalisée des mouvements de chaque enfant en relation avec les mouvements de l'objet. Ces traces graphiques sont exploitées par les chercheurs en psychopathologies afin d'évaluer l'efficacité de l'entraînement à la collaboration motrice à l'aide du dispositif et également par les praticiens qui peuvent mettre en place des 

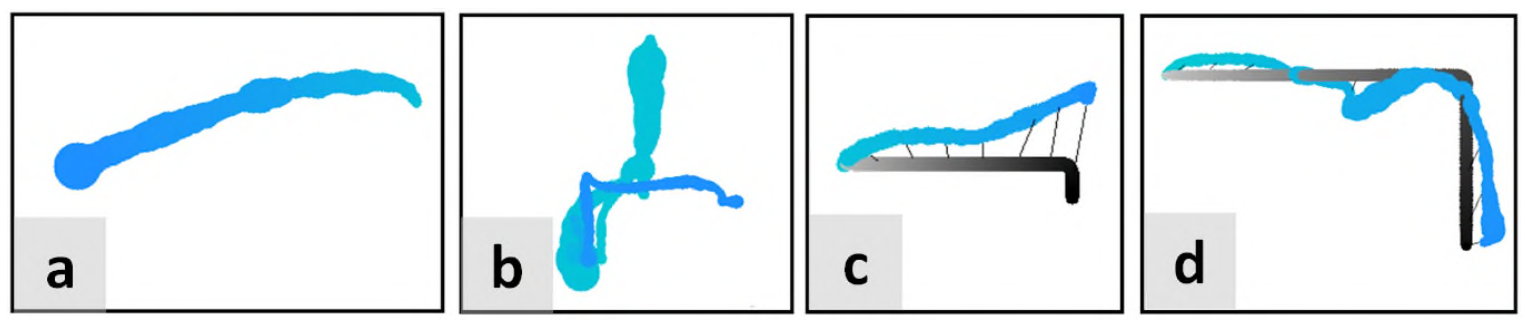

Figure 8: différentes visualisations des trajectoires d'un enfant avec l'agent suiveur (a et b) et avec l'agent meneur (c et d). a. Le mouvement de l'enfant accélère progressivement. b. L'enfant explore en commençant par monter l'objet très haut avant de revenir à la position initiale et d'effectuer la trajectoire demandée. c. l'enfant s'éloigne progressivement de l'agent. d. l'enfant suit l'agent et adapte son mouvement pour rester aligné avec lui.

thérapies pour lesquelles il est précieux de revoir à posteriori les séances.

\section{ETUDE D'USAGE PRELEMINAIRE}

Dans cette section, nous rendons compte de manière qualitative de notre première étude, conduite avec des enfants typiques n'étant pas atteints de TSA. Cette étude qualitative a pour objectif de recueillir les premiers retours sur l'utilisabilité du système par des enfants. L'objectif n'est pas de tester la capacité du système à entraîner les enfants à réaliser des actions conjointes mais plutôt de tester leur compréhension de la fenêtre réelle-virtuelle et des actions à réaliser en collaboration avec l'agent. En parallèle, cette étude a pour objectif de nous permettre de détecter des problèmes ergonomiques et techniques. Dans cette section nous rapportons donc nos observations lors de ce test d'utilisabilité (pour lesquels il est généralement admis qu'entre 5 et 10 testeurs permettent d'identifier $80 \%$ des problèmes d'utilisabilité). Cette étude d'usage répondait à la nécessité de vérifier l'utilisabilité du dispositif pour de jeunes enfants capables de verbaliser leurs impressions et de concevoir son adaptabilité à différentes tailles et différentes statures de jeunes dans l'objectif de préparer un entraînement au long terme du système pour sa validation future avec des enfants porteurs de TSA. Un autre protocole expérimental avec enfants TSA est en cours (étude longitudinale et faits objectivés) et fera l'objet d'une autre publication.

\subsection{Participants}

Les enfants typiques sélectionnés se trouvent dans la même tranche d'âge que les enfants cibles (utilisateurs finaux), afin qu'ils aient des tailles et des poids similaires. La capacité des enfants typiques à verbaliser leurs impressions permet de recueillir des informations précieuses pour la conception du système. Dix enfants entre 4 et 10 ans (4 filles et 6 garçons) ont participé à cette étude. Leurs consentements ainsi que celui de leurs parents ont été recueillis comme requis par la déclaration d'Helsinki et le comité éthique de l'Université Paris-Saclay (le Comité d'Éthique de la Recherche a donné un avis favorable à l'étude).

\subsection{Procédure}

Dès leur arrivée, les parents prennent connaissances et signent (s'ils le souhaitent) un formulaire de consentement, accompagné d'une description de nos objectifs de recherche ainsi que des tâches demandées à leur enfant. Une attention particulière a été portée à la compréhension par les parents et les enfants que ce n'étaient pas l'enfant qui était testé mais bien le système. Les enfants étaient encouragés à exprimer leurs impressions, bonnes et/ou mauvaises, à tout moment. L'enfant réalisait les tâches, guidé par un expérimentateur, pendant que deux autres expérimentateurs restaient en tant qu'observateurs, les parents restant présents dans la pièce. L'interaction avec le système durait environ 15 minutes, suivi d'une interview semi-structurée (grille d'entretien en annexe) d'une durée de 20 minutes. L'interview était menée autour de l'appréciation du système, de la compréhension de la fenêtre réelle-virtuelle, de l'interaction avec les objets et le mur et enfin sur l'action conjointe avec l'agent. Les parents étaient encouragés à intervenir durant l'interview pour éventuellement reformuler et clarifier les questions. Les expérimentateurs prenaient leurs notes d'observation et d'entretien à même ce guide. Pour résumer, pour chaque enfant les données collectées étaient composées de son âge, son poids, sa taille et les notes des trois expérimentateurs. Pour l'analyse, les notes des trois expérimentateurs ont ensuite été croisées.

Lors de la tâche, chaque enfant devait réaliser une série de mouvements avec le système, et ce dans 3 conditions différentes : sans agent, avec l'agent suiveur et avec l'agent meneur. Ces mouvements étaient :

- Déplacer la boîte bleue depuis le sol vers le dessus du tabouret rouge

- Déplacer la boîte bleue depuis le dessus du tabouret rouge vers le dessus de la table violette

- Déplacer la boîte bleue depuis le dessus de la table violette vers le dessus du tabouret rouge

- Déplacer la boîte bleue depuis le dessus du tabouret rouge vers le sol

\subsection{Résultats préliminaires}

5.3.1 Appréciation. Globalement, le système a été bien reçu, en particulier lorsque les personnages faisaient leur apparition. Le fait de les appeler par leur nom, Lola et Michou, a généré de la curiosité et des réactions amusées chez les enfants. Bien que nous n'ayons pas observé de réactions négatives ou de frustration, les enfants plus âgés ont montré une baisse d'excitation vers la fin de leur séance, surement dû au fait qu'ils accomplissaient les tâches très facilement. Ces enfants typiques comprenaient bien la différence entre un "succès" (étoile) et un "échec" (fumée). 
5.3.2 Fenêtre réelle-virtuelle. Bien que certains enfants aient noté un mauvais alignement entre l'objet réel et l'objet virtuel, l'ensemble des enfants semble avoir perçu que l'espace virtuel se trouvait "en face d'eux". Il n'y a pas eu de remarques négatives sur la qualité de l'affichage ou sur d'éventuels difficultés à percevoir le monde virtuel. Lorsque les enfants détachaient de façon accidentelle l'objet du mur, ils comprenaient comment remettre l'objet en place. Il y avait parfois un peu d'approximation au niveau de l'alignement requis cependant. Un bug apparu à deux reprises (donc avec un impact limité sur l'expérimentation) déclenchait des mouvements en décalage de la partie virtuelle, cassant le lien virtuel-réel, et menant l'objet virtuel à sortir de l'écran. Les enfants percevaient l'incohérence de la situation et se mettaient à rire. Le seul moment où les enfants se sont éloignés de la structure, comme pour indiquer une rupture de l'immersion dans l'interaction, fut lorsqu'il a été nécessaire de retourner au menu pour changer de mode d'interaction, comme prévu par notre protocole.

5.3.3 Interaction avec le mur. Tous les enfants ont spontanément détaché l'objet du mur la première fois. Naturellement, ils cherchent d'abord à lever et tirer l'objet, le faire glisser sur la paroi ne semblant pas intuitif. Les enfants ont ainsi dû s'adapter pour utiliser le système et cette adaptation a pris plus ou moins de temps en fonction des enfants (certains l'ont assimilé immédiatement lors du 2ème essai, d'autres ont eu besoin de 5 minutes). Nous avons également pu noter que déplacer l'objet lorsqu'il est situé plus bas que les genoux semblait être plus difficile. Bien que tous les enfants aient pu surmonter leur difficulté et terminer l'expérience, certains ont dû parfois changer de posture pour faire glisser l'objet, la résistance semblant un peu forte pour eux (pour les plus jeunes, 4-5 ans). Néanmoins, cette difficulté leur est apparue naturelle au vu du scénario qui consiste à déplacer à deux un objet lourd.

5.3.4 Perception de l'action conjointe. Lorsque nous leur avons demandé de décrire l'interaction, les enfants ont répondu qu'ils avaient déplacé l'objet avec Lola ou Michou, leur donnant ainsi vie. Le mouvement de saisie de l'objet virtuel par l'agent a déclenché à plusieurs reprises un mouvement de saisie de l'objet réel chez certains enfants. Deux enfants ont cependant décrit la tâche de suivi de Lola (l'agent meneur) comme un déplacement en même temps qu'elle, la percevant plutôt comme une action en parallèle que comme une action conjointe.

\subsection{Corrections du système}

Grâce à cette étude, nous avons recueilli des retours précieux sur un ensemble d'éléments pouvant être modifiés pour faciliter le scénario d'entraînement d'enfants atteints de TSA :

- Faciliter le déplacement de l'objet : déplacer un objet selon un plan en deux dimensions n'est pas naturel pour un enfant et il est important de familiariser les enfants avec le mode de déplacement de l'objet. Par conséquent, il est plus pertinent de faire référence au mouvement comme un glissement plutôt que comme un déplacement ou un mouvement.

- Adapter la hauteur minimale de l'étagère du bas : les enfants les plus grands ont eu des difficultés à déplacer l'objet lorsqu'il était bas. Nous avons donc conçu une étagère ajustable permettant de régler la hauteur minimale de la zone d'interaction.

- Limiter l'impact de l'écran d'accueil : la rupture d'immersion provoquée par le retour au menu principal nous a conduit à changer celui-ci afin qu'il soit présenté comme une vue sur l'environnement virtuel et ses objets, afin de préserver la continuer réelle-virtuelle.

- Éviter les distractions dues à des feedbacks pour les praticiens : les enfants ont eu des remarques sur les éléments d'interfaces à destination des praticiens. Nous avons donc fait en sorte de les rendre moins visibles en retirant leur couleur, en ajoutant de la transparence et en diminuant leur taille.

\section{DISCUSSION}

Avec cet article, nous avons présenté un système interactif permettant l'entraînement à l'action conjointe, entraînement à la fois des compétences motrices et sociales, pour des enfants atteints de TSA. La conception d'un tel système virtuel et tangible est l'aboutissement d'une approche pluridisciplinaire intégrant psychopathologie, conception mécanique, développement logiciel et prise en compte des utilisateurs. Le principal intérêt d'une telle thérapie est qu'en introduisant du social au sein d'un entraînement moteur, on rend accessible une thérapie sociale à des enfants atteints de TSA et non-verbaux. D'un autre côté, le principal enjeu d'un tel système est qu'en cherchant à simuler la co-manipulation physique, la plupart des dispositifs robotiques capables de le faire ne remplissent pas les contraintes d'innocuités qu'un environnement aussi sensible qu'un lieu d'accueil pour enfants atteints de TSA requiert. Aussi, la proposition originale de ce projet de recherche est de s'écarter de la piste robotique pour explorer celle de l'humain virtuel augmenté. Le système est composé d'un personnage virtuel projeté sur une surface verticale sur laquelle un objet tangible est magnétisé : l'enfant et le personnage virtuel tiennent et déplacent tous deux l'objet, ce qui permet de simuler une action conjointe. Le mur projeté est une fenêtre sur l'espace virtuel du personnage virtuel supposé être la continuité de l'espace réel du participant, les participants peuvent "voir à travers" le mur. À notre connaissance, ce système interactif est le premier à proposer un tel entraînement d'action conjointe adaptée aux jeunes enfants atteints de TSA. Les premiers résultats d'une étude de faisabilité avec enfants typiques ont montré le potentiel du système : le dispositif interactif a été accepté et perçu comme attrayant, le paradigme interactif de la fenêtre réelle-virtuelle a fonctionné et les enfants ont compris là tâches du déplacement à deux d'objets à la fois tangibles et virtuels.

Poussés par la forte contrainte de concevoir un système inoffensif et visuellement minimaliste, nous avons développé un dispositif dans lequel toutes les technologies nécessaires sont intégrées derrière un mur, contraignant l'interaction à un plan 2D. Avec le système proposé, déplacer un objet avec l'agent virtuel implique de comprendre et d'être capable de deux choses : les objets tangibles doivent être déplacés en les faisant glisser le long du mur, et ce mouvement de glissement doit se faire tout en maintenant la partie tangible aimantée à la partie arrière (c'est-à-dire en restant aligné avec la partie virtuelle). Ces contraintes d'interaction n'ont pas été évidentes pour tous les enfants de l'étude : certains enfants, 
pour compenser leur tendance naturelle à tirer en même temps que pousser l'objet, s'appuyaient de tout leur corps sur l'objet en mousse à déplacer (nous parlons ici d'un enfant de 4 ans). Bien que l'ensemble des enfants aient apprécié l'interaction et aient considéré comme «normal »la difficulté de déplacement de l'objet, nous pouvons nous poser la question de la transférabilité à la vie réelle des compétences développées lors d'une telle tâche. Une piste à développer pour limiter l'écart entre la tâche au sein du système et une tâche dans la vie réelle serait de diminuer davantage la friction entre l'objet tangible et le mur : moins de friction permettra notamment d'augmenter la puissance des aimants et donc de diminuer la tendance naturelle à détacher les objets du mur.

Enfin, il est important de noter que le dispositif proposé a été développé dans l'objectif de fournir cet entraînement et ce suivi personnalisé à des enfants atteints de TSA. Il est cependant nécessaire de tester le dispositif en amont sur un public intermédiaire, moins sensible. Dans cet article, l'étude proposée suit cet objectif : elle rapporte les retours pour une interaction avec des enfants typiques du même âge chronologique que la population cible. Si les retours sont importants pour valider à minima le paradigme d'interaction (perceptivement et physiquement), il n'en reste pas moins que nous ne savons pas comment le dispositif sera reçu par une population nécessairement hétérogène d'enfants atteints de TSA. Les phases suivantes du projet permettront de mettre en place des entraînements avec ces populations cibles en prenant en compte l'écosystème global dans lequel évoluent les enfants avec TSA [47].

\section{REMERCIEMENTS}

Ce projet a été financé dans le cadre de l'appel à projets «Autisme et Nouvelles Technologies », coordonné par la FIRAH et soutenu par la fondation UEFA pour enfants, et la fondation Orange. Logiciel pour l'entrainement combine a l'interaction sociale coopérative et a l'apprentissage moteur : https://www.firah.org/logiciel-pour-1entrainement-combine-a-1-interaction-sociale-cooperative-et-a1-apprentissage-moteur.html.

\section{RÉFÉRENCES}

[1] M. J. Richardson, K. L. Marsh, et R. M. Baron, «Judging and actualizing intrapersonal and interpersonal affordances », Journal of Experimental Psychology: Human Perception and Performance, vol. 33, no 4, p. 845-859, 2007, doi: 10.1037/00961523.33.4.845.

[2] R. C. Schmidt et M. J. Richardson, «Dynamics of Interpersonal Coordination », in Coordination: Neural, Behavioral and Social Dynamics, A. Fuchs et V. K. Jirsa, Éd. Berlin, Heidelberg: Springer, 2008, p. 281-308.

[3] J. Nadel, «Perception-action coupling and imitation in autism spectrum disorder », Developmental Medicine \& Child Neurology, vol. 57, no s2, p. 55-58, 2015, doi: 10.1111/dmcn.12689.

[4] O. Grynszpan, P. L. (Tamar) Weiss, F. Perez-Diaz, et E. Gal, «Innovative technology-based interventions for autism spectrum disorders: A meta-analysis », Autism, vol. 18, no 4, p. 346-361, mai 2014, doi: 10.1177/1362361313476767.

[5] B. O. Ploog, A. Scharf, D. Nelson, et P. J. Brooks, «Use of Computer-Assisted Technologies (CAT) to enhance social, communicative, and language development in children with autism spectrum disorders », Journal of Autism and Developmental Disorders, vol. 43, no 2, p. 301-322, 2013, doi: 10.1007/s10803-012-1571-3.

[6] S. Provoost, H. M. Lau, J. Ruwaard, et H. Riper, «Embodied Conversational Agents in Clinical Psychology: A Scoping Review », Journal of Medical Internet Research, vol. 19, no 5, p. e151, 2017, doi: 10.2196/jmir.6553.

[7] G. A. Francis, W. Farr, S. Mareva, et J. L. Gibson, «Do Tangible User Interfaces promote social behaviour during free play? A comparison of autistic and typically-developing children playing with passive and digital construction toys », Research in Autism Spectrum Disorders, vol. 58, p. 68-82, févr. 2019 , doi: 10.1016/j.rasd.2018.08.005.

[8] E. Hornecker et J. Buur, «Getting a grip on tangible interaction: a framework on physical space and social interaction », in Proceedings of the SIGCHI Conference on Human Factors in Computing Systems, Montréal, Québec, Canada, avr. 2006, p. 437-446, doi: 10.1145/1124772.1124838.

[9] V. Tam, M. Gelsomini, et F. Garzotto, «Polipo: a Tangible Toy for Children with Neurodevelopmental Disorders », in Proceedings of the Eleventh International Conference on Tangible, Embedded, and Embodied Interaction, Yokohama, Japan, mars 2017, p. 11-20, doi: 10.1145/3024969.3025006.

[10] A. I. Soysa et A. Al Mahmud, «Tangible Play and Children with ASD in LowResource Countries: A Case Study », in Proceedings of the Fourteenth International Conference on Tangible, Embedded, and Embodied Interaction, Sydney NSW, Australia, févr. 2020, p. 219-225, doi: 10.1145/3374920.3374951.

[11] D. B. Legoff et M. Sherman, «Long-term outcome of social skills intervention based on interactive LEGO๑play », Autism, vol. 10, no 4, p. 317-329, juill. 2006, doi: 10.1177/1362361306064403.

[12] W. Farr, N. Yuill, et H. Raffle, «Social benefits of a tangible user interface for children with Autistic Spectrum Conditions », Autism, vol. 14, no 3, p. 237-252, mai 2010, doi: 10.1177/1362361310363280.

[13] W. Farr, N. Yuill, et S. Hinske, «An augmented toy and social interaction in children with autism », IJART, vol. 5, p. 104-125, 2012, doi: 10.1504/ijart.2012.046270.

[14] S. Jordà, G. Geiger, M. Alonso, et M. Kaltenbrunner, «The reacTable: Exploring the Synergy Between Live Music Performance and Tabletop Tangible Interfaces ", in Proceedings of the 1st International Conference on Tangible and Embedded Interaction, New York, NY, USA, 2007, p. 139-146, doi: 10.1145/1226969.1226998.

[15] A. M. Piper, E. O’Brien, M. R. Morris, et T. Winograd, «SIDES: A Cooperative Tabletop Computer Game for Social Skills Development », in Proceedings of the 2006 20th Anniversary Conference on Computer Supported Cooperative Work, New York, NY, USA, 2006, p. 1-10, doi: 10.1145/1180875.1180877.

[16] E. Gal et al., «Enhancing social communication of children with high-functioning autism through a co-located interface », AI \& Soc, vol. 24, no 1, p. 75, févr. 2009, doi: 10.1007/s00146-009-0199-0.

[17] A. Battocchi et al., «Collaborative puzzle game: a tabletop interface for fostering collaborative skills in children with autism spectrum disorders », Journal of Assistive Technologies, vol. 4, no 1, p. 4-13, janv. 2010, doi: 10.5042/jat.2010.0040.

[18] L. Giusti, M. Zancanaro, E. Gal, et P. L. (Tamar) Weiss, «Dimensions of Collaboration on a Tabletop Interface for Children with Autism Spectrum Disorder », in Proceedings of the SIGCHI Conference on Human Factors in Computing Systems, New York, NY, USA, 2011, p. 3295-3304, doi: 10.1145/1978942.1979431.

[19] M. Jouaiti et P. Hénaff, «Robot-Based Motor Rehabilitation in Autism: A Systematic Review », Int J of Soc Robotics, vol. 11, no 5, p. 753-764, déc. 2019, doi: 10.1007/s12369-019-00598-9.

[20] K. Dautenhahn et A. Billard, «Games Children with Autism Can Play with Robota, a Humanoid Robotic Doll », in Universal Access and Assistive Technology, London, 2002, p. 179-190, doi: 10.1007/978-1-4471-3719-1_18.

[21] Z. Zheng, E. M. Young, A. R. Swanson, A. S. Weitlauf, Z. E. Warren, et N. Sarkar, «Robot-Mediated Imitation Skill Training for Children With Autism », IEEE Transactions on Neural Systems and Rehabilitation Engineering, vol. 24, no 6, p. 682-691, juin 2016, doi: 10.1109/TNSRE.2015.2475724.

[22] A. Billard, B. Robins, J. Nadel, et K. Dautenhahn, «Building Robota, a MiniHumanoid Robot for the Rehabilitation of Children with Autism », Assistive Technology, vol. 19, no 1, p. 37-49, 2007.

[23] A. Duquette, F. Michaud, et H. Mercier, «Exploring the use of a mobile robot as an imitation agent with children with low-functioning autism », Auton Robot, vol. 24, no 2, p. 147-157, févr. 2008, doi: 10.1007/s10514-007-9056-5.

[24] S. Ali et al., «An Adaptive Multi-Robot Therapy for Improving Joint Attention and Imitation of ASD Children », IEEE Access, vol. 7, p. 81808-81825, 2019, doi: 10.1109/ACCESS.2019.2923678.

[25] S. Serret et al., «Facing the challenge of teaching emotions to individuals with low- and high-functioning autism using a new Serious game: a pilot study », Molecular Autism, vol. 5, no 1, p. 37, juill. 2014, doi: 10.1186/2040-2392-5-37.

[26] C. Grossard et al., «Teaching Facial Expression Production in Autism: The Serious Game JEMImE », Creative Education, vol. 10, no 11, p. 2347, nov. 2019, doi: 10.4236/ce.2019.1011167.

[27] M. Courgeon, G. Rautureau, J.-C. Martin, et O. Grynszpan, «Joint Attention Simulation Using Eye-Tracking and Virtual Humans », IEEE Transactions on Affective Computing, vol. 5, no 3, p. 238-250, juill. 2014, doi: 10.1109/TAFFC.2014.2335740.

[28] S. Bernardini, K. Porayska-Pomsta, et T. J. Smith, «ECHOES: An intelligent serious game for fostering social communication in children with autism », Information Sciences, vol. 264, p. 41-60, avr. 2014, doi: 10.1016/j.ins.2013.10.027.

[29] E. Marchi et al., «The ASC-Inclusion Perceptual Serious Gaming Platform for Autistic Children », IEEE Transactions on Games, vol. 11, no 4, p. 328-339, déc. 2019, doi: 10.1109/TG.2018.2864640.

[30] C. Grossard, O. Grynspan, S. Serret, A.-L. Jouen, K. Bailly, et D. Cohen, «Serious games to teach social interactions and emotions to individuals with autism spectrum disorders (ASD) », Computers \& Education, vol. 113, p. 195-211, oct. 2017, doi: 10.1016/j.compedu.2017.05.002.

[31] D. A. Trevisan, J. T. Enns, E. Birmingham, et G. Iarocci, «Action coordination during a real-world task: Evidence from children with and without autism spectrum disorder », Development and Psychopathology, p. 1-11, undefined/ed, doi: $10.1017 /$ S0954579419001561. 
[32] J. Stückler et S. Behnke, «Following human guidance to cooperatively carry a large object », in 2011 11th IEEE-RAS International Conference on Humanoid Robots, oct. 2011, p. 218-223, doi: 10.1109/Humanoids.2011.6100917.

[33] N. Jarrassé, V. Sanguineti, et E. Burdet, «Slaves no longer: review on role assign ment for human-robot joint motor action », Adaptive Behavior, vol. 22, no 1, p. 70-82, févr. 2014, doi: 10.1177/1059712313481044.

[34] D. J. Agravante, A. Cherubini, A. Sherikov, P. Wieber, et A. Kheddar, «HumanHumanoid Collaborative Carrying », IEEE Transactions on Robotics, vol. 35, no 4, p. 833-846, août 2019, doi: 10.1109/TRO.2019.2914350.

[35] J. Saldien, K. Goris, B. Vanderborght, J. Vanderfaeillie, et D. Lefeber, «Expressing emotions with the social robot Probo », INTERNATIONAL JOURNAL OF SOCIAL ROBOTICS, vol. 2, no 4, Art. no 4, 2010, doi: 10.1007/s12369-010-0067-6.

[36] K. Goris, J. Saldien, B. Vanderborght, et D. Lefeber, «Mechanical design of the huggable robot probo », Int. J. Human. Robot., vol. 08, no 03, p. 481-511, sept 2011, doi: 10.1142/S0219843611002563.

[37] R. E. Simut, J. Vanderfaeillie, A. Peca, G. Van de Perre, et B. Vanderborght, «Chil dren with Autism Spectrum Disorders Make a Fruit Salad with Probo, the Social Robot: An Interaction Study », J Autism Dev Disord, vol. 46, no 1, p. 113-126, janv. 2016, doi: 10.1007/s10803-015-2556-9.

[38] D. Casas-Bocanegra et al., «An Open-Source Social Robot Based on Compliant Soft Robotics for Therapy with Children with ASD », Actuators, vol. 9, no 3, Art no 3, sept. 2020, doi: 10.3390/act9030091.

[39] M. Lee et al., «The wobbly table: Increased social presence via subtle incidental movement of a real-virtual table », in 2016 IEEE Virtual Reality (VR), mars 2016, p. 11-17, doi: 10.1109/VR.2016.7504683.

[40] F. Pa, P. X, et de C. H. Af, «Reduced Mimicry to Virtual Reality Avatars in Autism Spectrum Disorder. », J Autism Dev Disord, vol. 46, no 12, p. 3788-3797, déc. 2016, doi: 10.1007/s10803-016-2930-2

[41] A. Tartaro, J. Cassell, C. Ratz, J. Lira, et V. Nanclares-Nogués, «Accessing Peer Social Interaction », ACM Transactions on Accessible Computing (TACCESS), déc. 2014, Consulté le: déc. 29, 2019. [En ligne]. Disponible sur: https://dl.acm. org/doi/abs/10.1145/2700434.

[42] G. Herrera et al., «Pictogram Room: Natural Interaction Technologies to Aid in the Development of Children with Autism », Annuary of Clinical and Health Psychology, vol. 08, p. 39-44, janv. 2012.

[43] G. Herrera, P. Pérez-Fuster, et G. Poli, «Pictogram Room: son efficacité dans le trouble du spectre de l'autisme (TSA) », Enfance, vol. $\left.\mathrm{N}_{\{\circ}^{\circ}\right\}$ 1, no 1, p. 31-50, mai 2018.

[44] J. Nadel, How Imitation Boosts Development: In Infancy and Autism Spectrum Disorder. Oxford University Press, 2014

[45] L. Benton, A. Vasalou, R. Khaled, H. Johnson, et D. Gooch, «Diversity for design: a framework for involving neurodiverse children in the technology design process », in Proceedings of the SIGCHI Conference on Human Factors in Computing Systems, Toronto, Ontario, Canada, avr. 2014, p. 3747-3756, doi: $10.1145 / 2556288.2557244$.

[46] K. Kim, R. Schubert, et G. Welch, «Exploring the Impact of Environmental Effects on Social Presence with a Virtual Human », in Intelligent Virtual Agents, Cham, 2016, p. 470-474, doi: 10.1007/978-3-319-47665-0 57.

[47] M. Guffroy. Adaptation de méthodes d'évaluation dans le cadre de la conception d'une application numérique pour un jeune public avec troubles du spectre autistique : étude au cours de la conception et de l'évaluation de l'application çATED au sein d'une ULIS TED. Environnements Informatiques pour l'Apprentissage Humain. Université du Maine, 2017.

\section{A A ANNEXE: GUIDE D'ENTRETIEN}

Liste des questions par thématiques posées lors de l'entretien semistructuré:

- Appréciation du dispositive:

- Peut-être pour commencer, est-ce que l'interaction t'a plu?

- Si oui, qu'est-ce qui t'as plu?
- L'objet, le personnage, le jeu...

- Quelque chose qui t'aurait amusé ou intéressé ?

- Est-ce qu'il y a des choses qui t'ont déplu?

- L'objet, le personnage, le jeu...

- Rien ne t'a effrayé ou énervé par exemple?

- Est-ce qu'il y a des choses que tu n'as pas comprises ?

- Est-ce que ce jeu te fait penser à d'autres jeux ?

- Si tu pouvais améliorer le jeu, qu'ajouterais-tu?

- Transition réel-virtuel:

- Pourrais-tu me raconter avec tes mots en quoi consistait le jeu que tu viens de faire?

- Pour toi, le personnage virtuel, il se trouvait loin de toi ?

- Loin comment ? Il serait où dans la pièce?

- L'objet que tu as déplacé, c'est quoi comme objet d'après toi ?

- Et l'objet dans l'image, était-il différent d'après toi ?

- Si l'enfant a détaché l'objet du mur : peux-tu me décrire ce qui s'est passé quand tu as détaché l'objet du mur?

- Tu as pensé quoi sur le moment?

- Si l'enfant n'a pas détaché l'objet du mur : as-tu eu m'envie de détacher l'objet du mur?

- Pourquoi ?

- Si tu veux tu peux le détacher maintenant! (ici poser les deux questions du dessus)

- Facilité de déplacement de l'objet:

- As-tu trouvé difficile de déplacer l'objet?

- Trop dur, ou trop bizarre comme mouvement

- Est-ce qu'il y a des mouvements qui étaient plus difficiles que d'autres à faire?

- Peux-tu m'expliquer?

- Est-ce qu'il y a des mouvements que tu aurais aimé pouvoir faire avec l'objet?

- D'après toi, est-ce que l'objet était lourd ?

- Lourd comment? Tu peux me donner un exemple?

- Est-ce que tu dirais que porter cet objet c'est comme porter un objet dans la vraie vie?

- Peux-tu m'expliquer la différence?

- Perception de l'action conjointe:

- D'après toi, le personnage virtuel tenait l'objet avec toi ?

- D'après toi, est-ce que le personnage virtuel faisait un effort pour porter l'objet?

- As-tu senti qu'il t'aidait ? Ou était-ce toi qui l'aidait ?

- Est-ce que tu crois que le personnage virtuel savait où il fallait déplacer l'objet?

- Penses-tu que tu aurais pu faire le jeu sans le personnage virtuel?

- Est-ce que cela aurait été différent pour toi ? 